\title{
Comparison of ACIOL Exchange and Descemet Membrane Endothelial Keratoplasty with ACIOL Retention and Descemet Stripping Automated Endothelial Keratoplasty in the Setting of Pseudophakic Bullous Keratopathy
}

Biana Dubinsky-Pertzov

Yitzhak Shamir Medical Center Assaf Harofeh

\section{Gissela Santaella}

University of Toronto

Nir Sorkin

Tel Aviv Ichilov-Sourasky Medical Center: Tel Aviv Sourasky Medical Center

Lior Or

Yitzhak Shamir Medical Center Assaf Harofeh

Inbal Gazit

Yitzhak Shamir Medical Center Assaf Harofeh

Clara C. Chan

University of Toronto

David S. Rootman

University of Toronto

Adi Einan-Lifshitz ( $\square$ adi.einan@gmail.com )

Yitzhak Shamir Medical Center Assaf Harofeh

\section{Research Article}

Keywords: ACIOL, PBK, DMEK, DSAEK, Intraocular lens

Posted Date: May 17th, 2021

DOI: https://doi.org/10.21203/rs.3.rs-499850/v1

License: (c) (i) This work is licensed under a Creative Commons Attribution 4.0 International License.

Read Full License 


\section{Abstract}

Objective: To compare the clinical outcomes and complications of anterior chamber intraocular lens (ACIOL) exchange and Descemet membrane endothelial keratoplasty (DMEK) with ACIOL retention and Descemet stripping automated endothelial keratoplasty (DSAEK) in patients with PBK.

Methods: A multicenter retrospective cohort study. Patients with ACIOL who underwent endothelial keratoplasty procedure due to PBK between 2012-2018 in two tertiary medical centers, were identified. Clinical and demographical data including preoperative and postoperative characteristics were collected.

Results: Thirteen eyes in the "DMEK and ACIOL exchange" group and 15 in the "DSAEK and ACIOL retention" group were included in the analysis. Mean BCVA six months postoperatively was $0.51 \pm 0.20$ LogMAR (Snellen 20/64) and 0.57 \pm 0.22 LogMAR (Snellen 20/83) in the "DMEK and ACIOL exchange" group and "DSAEK and ACIOL retention" group, respectively $(P=0.38)$. Graft failure occurred in 6 eyes $(40 \%)$ in the "DSAEK and ACIOL retention" group; four of them were secondary failures occurring at an average follow-up time of $15 \pm 11.9$ months. In the "DMEK and ACIOL exchange" group, graft failure occurred in one eye and was a primary failure $(P=0.046)$. In the "DMEK and ACIOL exchange" group, postoperative complications were seen in 4 eyes (30.7\%). No postoperative complications were recorded in the "DSAEK and ACIOL retention" group $(P=0.035)$.

Conclusion: Despite the lower complication rate, the higher incidence of graft failure and the need for second keratoplasty in the DSAEK group along with the similar visual outcomes, might suggest that in the indication of $\mathrm{PBK}, \mathrm{ACIOL}$ exchange with DMEK offers a good alternative to $\mathrm{ACIOL}$ retention with DSAEK.

\section{Introduction}

Pseudophakic bullous keratopathy (PBK) in the presence of an anterior chamber intraocular lens (ACIOL) carries a challenge for endothelial keratoplasty (EK). In the planning of such surgery, two main issues need to be addressed - the retainment or extraction of the ACIOL and the type of EK. Due to its relative procedural simplicity and the ability to perform in the presence of ACIOL, Descemet stripping automated EK (DSAEK) with retention of the ACIOL served as the procedure of choice in complicated eyes such as those with ACIOL (1).

Descemet membrane EK (DMEK) in the setting of ACIOL poses a few challenges, including unfolding the DMEK scroll by tapping on the cornea (2), and DMEK graft tamponade in a unicameral eye (anterior vitrectomy is usually performed in eyes with $\mathrm{ACIOL}$ ). Although there are some publications describing DMEK in the presence of ACIOL, many surgeons prefer performing DSAEK in eyes with ACIOL.

However, in a study describing the results following DSAEK in eyes with PBK and a retained ACIOL, the graft survival and endothelial cell (EC) loss were significantly inferior compared with DSAEK in eyes with posterior chamber IOL, suggesting that retention of $\mathrm{ACIOL}$ leads to less desirable outcomes. Hence, 
removing the ACIOL might allow better results (3). Tannan et al. compared ACIOL retention and DSAEK with ACIOL exchange and DSAEK, and showed that IOL exchange was correlated with more intraoperative and postoperative complications (4).

Over the last years, DMEK has become the preferred procedure in the treatment of corneal endothelial dysfunction, demonstrating better visual outcomes, refractive results, and healing time with lower rejection rate and similar surgical hazards and EC loss (1,5-7). Combining DMEK with ACIOL exchange and IOL fixation to the sclera might be an acceptable alternative encompassing the advantages of DMEK without probable endothelial damage from the retained ACIOL.

To our knowledge, there are no publications comparing the outcomes of these two popular techniques: ACIOL exchange with scleral fixated IOL and DMEK with ACIOL retention and DSAEK. The aim of the proposed study was to compare the outcomes of these two techniques to optimize the management of PBK in the setting of ACIOL.

\section{Methods}

\section{Study design and population}

This is a multicentered retrospective cohort study. Patients who underwent ACIOL exchange and transscleral IOL fixation combined with DMEK or DSAEK with ACIOL retention due to PBK between 2012-2018 at Toronto Western Hospital, Canada and Shamir medical center, Israel were included. Patients were included if it was their first corneal transplantation surgery and had a minimum follow-up time of 6 months.

Between 2012-2015, the procedure of choice was DSAEK with ACIOL retention and starting from 2016, ACIOL exchange combined with DMEK became the common practice.

\section{Data collection}

Data extracted from patient charts included: demographic and corneal donor characteristics, bestcorrected visual acuity (BCVA) before and after surgery during all follow-up appointments (based on subjective refraction on each follow-up visit), intraoperative and postoperative complications and clinical outcomes including rebubble procedure and incidence of primary or secondary graft failure graft rejection, regarded as dichotomous variables.

\section{Primary and secondary outcomes}

Primary outcome was the incidence of graft failure in the two groups. Secondary outcomes included BCVA at six months postoperatively, incidence of graft rejection, and rebubble rate.

Primary graft failure was diagnosed when the cornea failed to clear three months postoperatively. Secondary failure was diagnosed when corneal edema developed after a period of corneal clarity. Graft rejection was diagnosed when otherwise unexplainable corneal edema developed or upon the new 
appearance of endothelial precipitates on the corneal graft. Follow-up time was calculated as the time passed from surgery until a diagnosis of graft failure or until the last documented visit.

\section{Ethical considerations}

This study adhered to the tenets of the Declaration of Helsinki and was approved by the Medical center's IRB in both sites.

\section{Surgical Technique}

Graft preparation was performed immediately prior to transplantation. Surgeries were performed using sub-tenon anesthesia.

\section{"DMEK and ACIOL exchange" group - ACIOL exchange with DMEK:}

Prior to the beginning of surgery, the DMEK graft was prepared by separating Descemet membrane from the stroma starting from the non-pigmented area of the trabecular meshwork 360 degrees . The stripping technique has been described previously by our group (8).

The ACIOL optic size was measured and a clear corneal incision was performed accordingly (usually 6 $\mathrm{mm}$ wide) with subsequent removal of the $\mathrm{ACIOL}$ and partial suturing of the corneal incision. A scleral fixation, using Hoffman pockets technique (9) was used to fixate the new IOL (MA30BA, AcrySof, Alcon). After removal of Descemet membrane, insertion of the DMEK graft into the anterior chamber using the Geuder injector (Heidelberg, Germany) was performed (10), and the tapping technique was used to unfold the graft (2)(10). After placing the graft in the correct orientation and location, an air bubble was inserted into the anterior chamber. BSS was then injected between the air bubble and the iris to deepen the anterior chamber, leaving an air bubble that is slightly larger than the graft's diameter.

\section{“DSAEK and ACIOL retention” group - DSAEK with ACIOL retention:}

DSAEK surgery was performed by a standardized technique as described previously $(11,12)$, using the Busin glide and pull-through technique (13).

\section{Statistical analysis}

Baseline and outcome variables were compared between the two groups. Incidence of graft failure was analyzed using the chi-square test for bivariate analysis. Survival analysis using the Kaplan-Meier curve was used to adjust for follow-up time. Continuous variables were analyzed using the Student T test or the Mann-Whitney U test according to their distribution. Normal distribution was evaluated with Q-Q plot, Shapiro-Wilk test, and histogram chart with a normal distribution curve. 
P-values beneath 0.05 on a two-sided test were considered statistically significant.

The IBM SPSS Statistics 25 (IBM SPSS Statistics for Windows, Version 25.0. Armonk, NY; IBM Corp) was used.

\section{Results}

Thirteen eyes of 13 patients in the "DMEK and ACIOL exchange" group and 15 eyes of 15 patients in the "DSAEK and ACIOL retention" group were included in the analysis. The patients did not differ significantly in their demographic and clinical preoperative characteristics (Table 1). Average follow-up time was 16.4 \pm 6.73 and $19.21 \pm 10.36$ months in the "DMEK and ACIOL exchange" group and the "DSAEK and ACIOL retention" group, respectively $(P=0.34)$.

Table 1

Preoperative demographic and clinical characteristics

\begin{tabular}{|c|c|c|c|}
\hline & $\begin{array}{l}\text { ACIOL exchange and } \\
\text { DMEK }(n=13)\end{array}$ & $\begin{array}{l}\text { ACIOL retention and } \\
\text { DSAEK }(n=15)\end{array}$ & $\begin{array}{l}P \\
\text { value }\end{array}$ \\
\hline Age, years (mean $\pm S D$ ) & $76.7 \pm 12.7$ & $76.8 \pm 11.2$ & 0.96 \\
\hline \multicolumn{4}{|l|}{ Gender } \\
\hline Female (\%) & $4(31 \%)$ & $5(33.3 \%)$ & 0.61 \\
\hline $\begin{array}{l}\text { Preoperative BCVA logMAR (mean } \pm \\
\text { SD) }\end{array}$ & $1.84 \pm 0.95$ & $1.97 \pm 0.82$ & 0.65 \\
\hline \multicolumn{4}{|l|}{ Ocular comorbidity } \\
\hline Glaucoma & $1(7.6 \%)$ & $3(20 \%)$ & 0.35 \\
\hline AMD & $2(15.4 \%)$ & $1(6.7 \%)$ & 0.44 \\
\hline \multicolumn{4}{|l|}{ Corneal graft characteristics } \\
\hline Donor age, years (mean \pm SD) & $65.3 \pm 4.7$ & $65.5 \pm 11.7$ & 0.17 \\
\hline Graft Diameter, mm (mean \pm SD) & 8.0 & $8.2 \pm 0.4$ & 0.18 \\
\hline $\begin{array}{l}\text { Time from harvest to } \\
\text { transplantation, days (mean } \pm S D \text { ) }\end{array}$ & $6.0 \pm 1.9$ & $6.1 \pm 2.7$ & 0.10 \\
\hline
\end{tabular}

Graft failure requiring another corneal transplant occurred in 6 eyes in the "DSAEK and ACIOL retention" group (40\%). Of those, four were secondary graft failures with an average follow-up time of $15 \pm 11.9$ (range 4-32) months to failure and the other two were primary graft failures. In the "DMEK and ACIOL exchange" group, primary graft failure occurred in one eye due to persistent hyphema $(7.7 \%)$ and there was no secondary graft failure during the follow-up time. Incidence of graft failure differed significantly 
between groups both in a bivariate analysis $(P=0.04)$ and when adjusting for follow-up time in a subgroup analysis of secondary failure (survival analysis $P=0.046$ ) (Fig. 1).

Graft detachment requiring rebubble was diagnosed in four eyes (30.8\%) in the "DMEK and ACIOL exchange" group at an average follow-up of 13.7 days (range 7-21 days). In all eyes, the graft reattached, and the cornea cleared up following one rebubble attempt. In the "DSAEK and ACIOL retention" group, rebubble was performed in three eyes at an average follow-up time of 10 days (range 120 days) after surgery. In two of the three eyes, the cornea failed to clear and resulted in primary graft failure. There was no between-groups difference in rebubble rate $(P=0.41)$.

BCVA improved significantly in both groups. In the "DMEK and ACIOL exchange" group, BCVA improved from $1.84 \pm 0.95$ (Snellen 20/1383, range 0.6-4) LogMAR prior to surgery to $0.51 \pm 0.20$ (Snellen 20/64, range 0.3-2) LogMAR six months after surgery $(p=0.01)$. In the "DSAEK and ACIOL retention" group, BCVA improved from $1.97 \pm 0.82$ (Snellen 20/1866, range 0.5-3) LogMAR prior to surgery to $0.57 \pm 0.22$ (Snellen 20/83, range $0.39-0.87)$ LogMAR six months after surgery $(P=0.027)$. Postoperative BCVA outcomes are presented in Fig. 2, no statistically significant difference was found between the groups.

An episode of graft rejection occurred in one eye $(7.7 \%)$ in the "DMEK and ACIOL exchange" group, and one eye (6.6\%) in the "DSAEK and ACIOL retention" group, 11 and 18 months after surgery respectively (P $=0.46)$. In both eyes, the rejection episode resolved after a course of topical steroids. Neither were diagnosed with graft failure during follow-up time.

In the "DMEK and ACIOL exchange" group, postoperative complications were seen in 4 eyes (30.7\%). Hyphema reported in two eyes immediately after surgery, macular edema reported three months postoperatively in one eye, and retinal detachment reported 22 days after surgery in one eye. No complications were recorded in the "DSAEK and ACIOL retention" group $(P=0.035)$.

\section{Discussion}

PBK in the setting of ACIOL presents a surgical dilemma for the cornea surgeon.

The decision regarding the optimal surgical management consists of two main components: first, whether to retain the ACIOL or to replace it with a sutured posterior chamber IOL, and second, to evaluate which type of EK is more suitable in this specific setting and would result in a better outcome.

On the one hand, retention of the ACIOL and coping only with the decompensated cornea is an easier and quicker procedure that could be performed under local anesthesia. However, ACIOL retention could cause a higher rate of postoperative EC loss and graft failure (14). As previously mentioned, the DMEK graft needs to be unfolded when inserted into the anterior chamber, unlike the DSAEK graft which is inserted already spread open. The presence of $\mathrm{ACIOL}$ makes this process more challenging and might cause excessive endothelial loss and higher rate of graft detachment with lower graft survival rate (15). Hence, in eyes with ACIOL retention, DSAEK might have a better outcome. 
Tannan et al. compared ACIOL retention and DSAEK with ACIOL exchange and DSAEK and found no difference in the incidence of graft rejection and primary or secondary graft failure (13). The question remaining unanswered is whether $\mathrm{ACIOL}$ exchange combined with DMEK results in better outcomes regarding graft survival, intraoperative and postoperative complications, and visual outcome compared with $\mathrm{ACIOL}$ retention and DSAEK.

Concerning graft survival, we found a significantly higher rates of graft failure (both primary and secondary) in the "DSAEK and ACIOL retention" group, compared to the "DMEK and ACIOL exchange" group, where ACIOL was removed and a new IOL fixated to the sclera. Since previous publications comparing primary DMEK to primary DSAEK have established that there is no significant difference in the ECD loss as a result of the procedure itself (1), the higher rate of secondary graft failure could be related to the retention of the $\mathrm{ACIOL}$ rather than to a different EK techniques.

Ang et al (3) showed that in eyes after DSAEK with retained ACIOLs, the EC loss three years postoperatively was significantly higher compared with eyes with posterior chamber IOL. Although ECD was not documented during follow-up time in our study, secondary failure is the clinical result of significant ECD loss $(16,17)$.

With these findings considered, we presume that the higher rate of secondary graft failure was a result of the retention of the ACIOL. Therefore, in younger patients with otherwise healthy eyes, we suggest considering $\mathrm{ACIOL}$ removal and fixation of the posterior chamber IOL rather than performing DSAEK surgery with retention of the ACIOL.

DMEK combined with ACIOL exchange and trans-scleral fixation is a more complicated procedure than DMEK or DSAEK alone as previously reported (18). Nevertheless, there was no statistically significant difference in primary graft failure, rebubble rate, or BCVA six months postoperatively between the groups.

The main disadvantage of DMEK with ACIOL exchange is a higher rate of complications compared with DSAEK and ACIOL retention in the immediate postoperative time and during the first three months after surgery. It has been reported that EK combined with ACIOL exchange is associated with a higher complication rate. In the mentioned above study by Tannan et al (19), it was reported that postoperative complications in which surgical or medical interventions were required, were more common in the ACIOL exchange group. This finding suggests that the higher complication rate in the "DMEK and ACIOL exchange" group in our study is a result of the removal of ACIOL and scleral fixation of the new IOL.

Considering the aforementioned assumptions that the ACIOL should be removed and a new IOL re-fixated and that the probable cause of the higher rate of complications is the process of IOL exchange itself, we now reach the final step in our pursuit of the preferred management of PBK in the setting of $A C I O L$, examining the type of EK. As mentioned above, the advantages of DMEK over DSAEK are widely reported(5-7). It is also known that in the setting of PBK the overall graft survival with either technique is lower than in other EK indications such as Fuchs' endothelial dystrophy. Nevertheless, graft survival after DMEK remains higher compared to DSAEK (20). As with other surgeries, we believe that "practice makes 
perfect" and since DMEK is a more challenging and complicated procedure, the impact of an experienced and skilled surgeons would be greater, resulting in better visual outcomes.

When assessing the patient prior to surgery, several parameters should be addressed before deciding on the type of procedure; age and presumable life expectancy, functional state, and ability to undergo general anesthesia or tolerate the discomfort of a longer procedure under local anesthesia. A younger patient with a relatively long lifespan is the most suitable candidate for ACIOL exchange with DMEK procedure, which provides a longer graft survival and probably improved visual outcome. Nonetheless, an older patient whose well-being is satisfying, can also benefit from this procedure, although longer and more complicated. Most importantly, these issues should be initially discussed with the patient. The patient should be presented with the higher risk of complications in the DMEK and ACIOL exchange procedure versus the higher rate of corneal graft failure in the DSAEK and ACIOL retention procedure. Above all, the procedure performed should be the one the surgeon is most comfortable with, in adherence to the principle of 'primum non nocere'.

Another matter to consider when approaching a decision regarding patients with $\mathrm{ACIOL}$ and PBK is the visual potential. Previous studies show that the visual improvement following these procedures, which are complicated to begin with, is limited. This finding coincides with the BCVA results in this study $(4,18)$.

Our study has several limitations. First, its retrospective nature and probable residual confounders. Second, the possible variability between surgeons in the different medical centers, although this was regarded in the statistical analysis. Third, the lack of endothelial cell count and the relatively small number of patients in each group.

In summary, ACIOL exchange combined with trans-scleral fixation of a posterior chamber IOL and DMEK for the treatment of PBK is a more complex procedure. Nevertheless, the higher rates of graft failure and the need for second keratoplasty with retainment of $A C I O L$ and DSAEK, might suggest that in this specific setting, ACIOL exchange with DMEK would provide a better alternative, especially in younger patients and eyes with poorly positioned ACIOL.

\section{References}

1. Deng SX, Lee WB, Hammersmith KM, Kuo AN, Li JY, Shen JF, et al. Descemet Membrane Endothelial Keratoplasty: Safety and Outcomes: A Report by the American Academy of Ophthalmology. Ophthalmology. 2018;125(2):295-310.

2. Yoeruek E, Bartz-Schmidt K-U. Novel Maneuver Facilitating Descemet Membrane Unfolding in the Anterior Chamber. Cornea. 2012;1.

3. Ang M, Li L, Chua D, Wong C, Htoon HM, Mehta JS, et al. Descemet's stripping automated endothelial keratoplasty with anterior chamber intraocular lenses: complications and 3-year outcomes. $\mathrm{Br} \mathrm{J}$ Ophthalmol. 2014 Aug;98(8):1028-32. 
4. Tannan A, Vo RC, Chen JL, Yu F, Deng SX, Aldave AJ. Comparison of ACIOL Retention With IOL Exchange in Patients Undergoing Descemet Stripping Automated Endothelial Keratoplasty. Cornea. 2015 Sep;34(9):1030-4.

5. Scorcia V, Matteoni S, Scorcia GB, Scorcia G, Busin M. Pentacam Assessment of Posterior Lamellar Grafts to Explain Hyperopization after Descemet's Stripping Automated Endothelial Keratoplasty. Ophthalmology. 2009 Sep 1;116(9):1651-5.

6. Turnbull AMJ, Tsatsos M, Hossain PN, Anderson DF. Determinants of visual quality after endothelial keratoplasty. Survey of Ophthalmology. 2016 May 1;61(3):257-71.

7. Hamzaoglu EC, Straiko MD, Mayko ZM, Sáles CS, Terry MA. The First 100 Eyes of Standardized Descemet Stripping Automated Endothelial Keratoplasty versus Standardized Descemet Membrane Endothelial Keratoplasty. Ophthalmology. 2015 Nov;122(11):2193-9.

8. Borovik AM, Perez M, Lifshitz T, Einan-Lifshitz A, Sorkin N, Boutin T, et al. Peripheral Blunt Dissection: Using a Microhoe-Facilitated Method for Descemet Membrane Endothelial Keratoplasty Donor Tissue Preparation. Cornea. 2017 Oct;36(10):1270-3.

9. Hoffman RS, Fine IH, Packer M. Scleral fixation without conjunctival dissection. Journal of Cataract \& Refractive Surgery. 2006 Nov;32(11):1907-12.

10. Sorkin N, Einan-Lifshitz A, Ashkenazy Z, Boutin T, Showail M, Borovik A, et al. Enhancing Descemet Membrane Endothelial Keratoplasty in Postvitrectomy Eyes With the Use of Pars Plana Infusion. Cornea. 2016;

11. Melles GRJ, Wijdh RHJ, Nieuwendaal CP. A technique to excise the descemet membrane from a recipient cornea (descemetorhexis). Cornea. 2004 Apr;23(3):286-8.

12. Terry MA, Shamie N, Chen ES, Hoar KL, Friend DJ. Endothelial keratoplasty a simplified technique to minimize graft dislocation, iatrogenic graft failure, and pupillary block. Ophthalmology. 2008 Jul;115(7):1179-86.

13. Aralikatti A, Dean S, Busin M, Shah S. Pull-through technique for graft insertion in DSAEK. Journal of Cataract \& Refractive Surgery. 2008 Mar;34(3):341.

14. Shah AK, Terry MA, Shamie N, Chen ES, Phillips PM, Hoar KL, et al. Complications and Clinical Outcomes of Descemet Stripping Automated Endothelial Keratoplasty With Intraocular Lens Exchange. American Journal of Ophthalmology. 2010 Mar 1;149(3):390-397.e1.

15. Droutsas K, Lazaridis A, Kymionis G, Chatzistefanou K, Papaconstantinou D, Sekundo W, et al. Endothelial keratoplasty in eyes with a retained angle-supported intraocular lens. Int Ophthalmol. 2019 May 1;39(5):1027-35.

16. Lass JH, Sugar A, Benetz BA, Beck RW, Dontchev M, Gal RL, et al. Endothelial Cell Density to Predict Endothelial Graft Failure After Penetrating Keratoplasty. Arch Ophthalmol. 2010 Jan 1;128(1):63-9.

17. Hjortdal J, Pedersen IB, Bak-Nielsen S, Ivarsen A. Graft rejection and graft failure after penetrating keratoplasty or posterior lamellar keratoplasty for fuchs endothelial dystrophy. Cornea. 2013 May;32(5):e60-63. 
18. Weller JM, Tourtas T, Kruse FE. Feasibility and Outcome of Descemet Membrane Endothelial Keratoplasty in Complex Anterior Segment and Vitreous Disease. Cornea. 2015 Nov;34(11):1351-7.

19. Tannan A, Vo RC, Chen JL, Yu F, Deng SX, Aldave AJ. Comparison of ACIOL Retention With IOL Exchange in Patients Undergoing Descemet Stripping Automated Endothelial Keratoplasty. Cornea. 2015 Sep;34(9):1030-4.

20. Woo J-H, Ang M, Htoon HM, Tan D. Descemet Membrane Endothelial Keratoplasty Versus Descemet Stripping Automated Endothelial Keratoplasty and Penetrating Keratoplasty. American Journal of Ophthalmology. 2019 Nov 1;207:288-303.

\section{Figures}

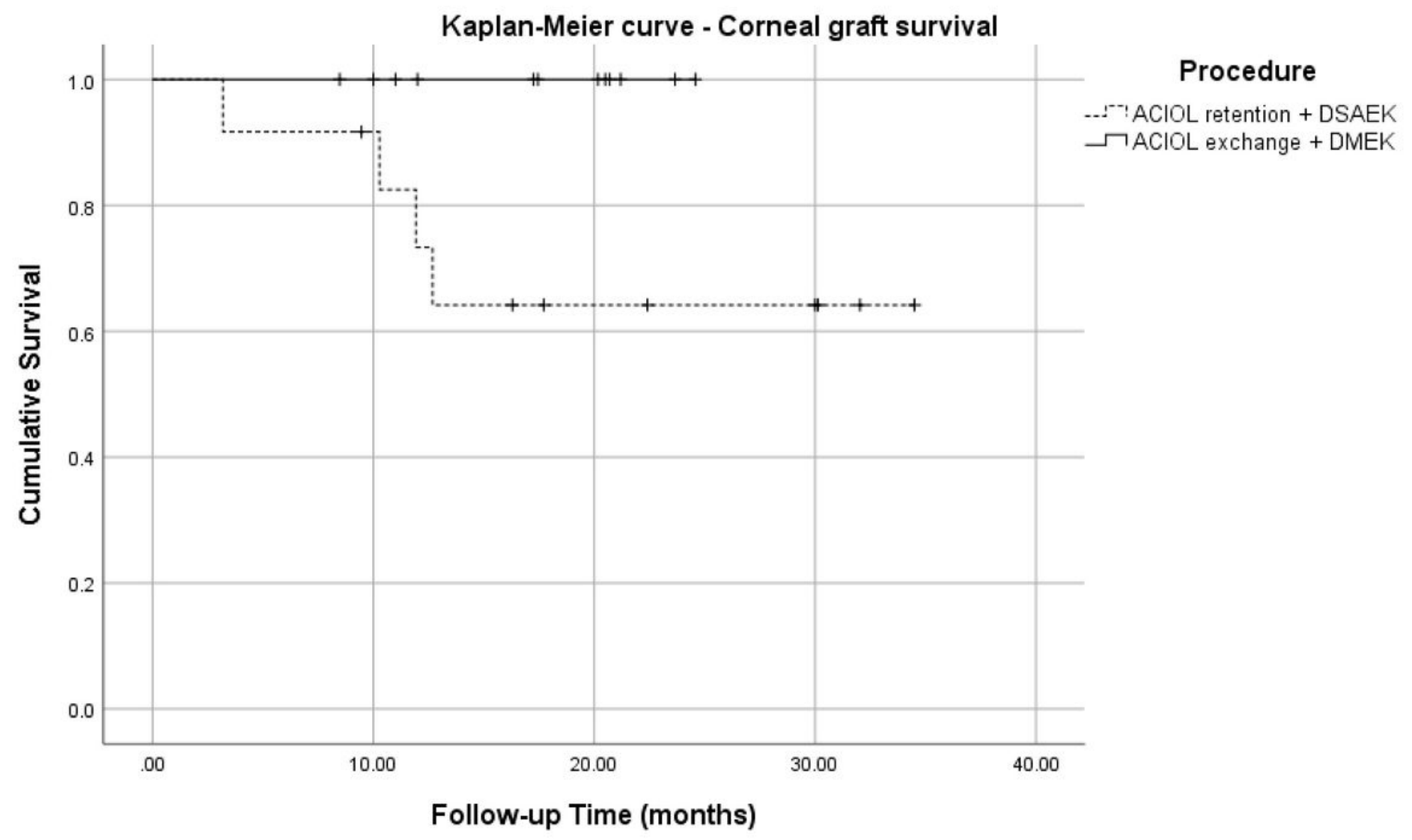

Figure 1

Kaplan-Meier curve of corneal graft survival in the two groups. 


\section{Postoperative BCVA}

1.20

1.00



0.20

0.00
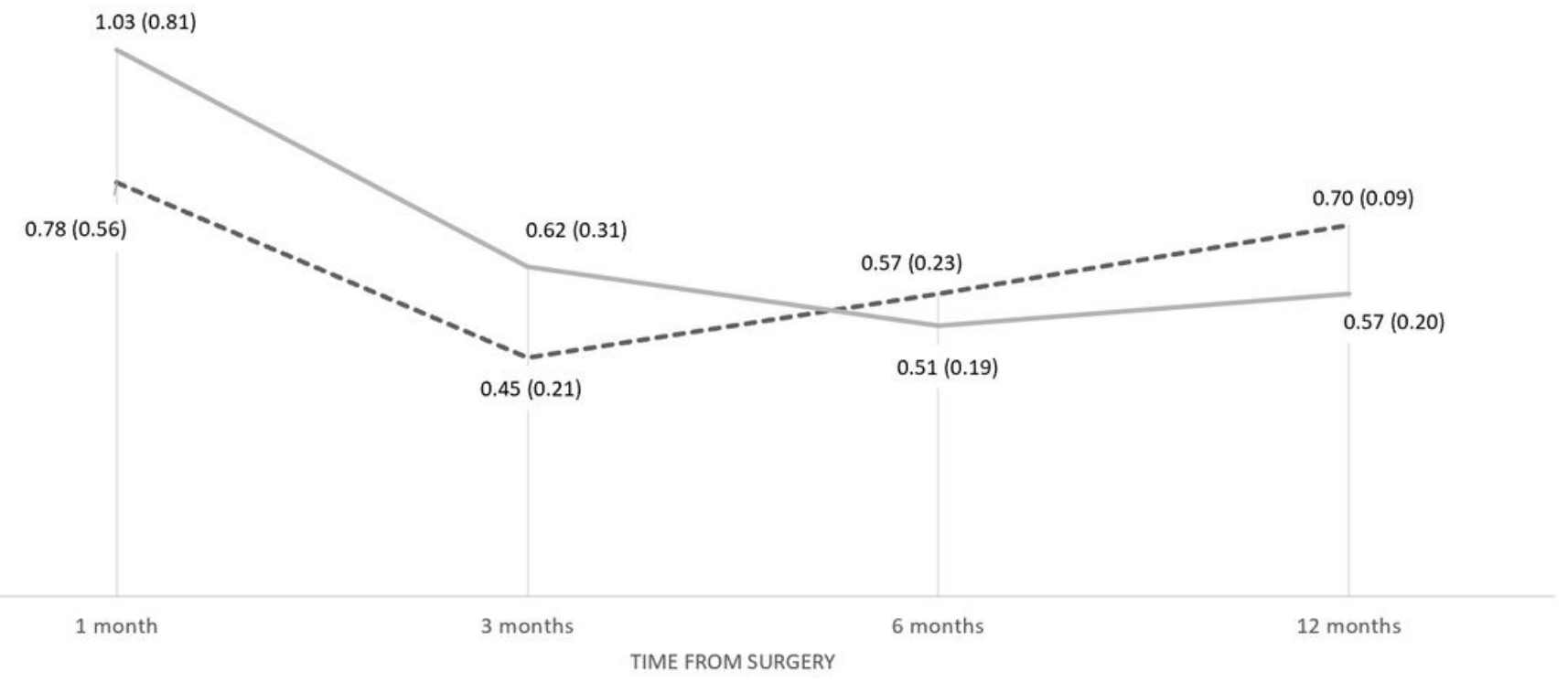

$---\mathrm{ACIOL}$ retention + DSAEK — ACIOL exchange + DMEK

\section{Figure 2}

Postoperative best corrected visual acuity in the two groups on each follow-up visit after surgery; Mean (SD). The visual outcomes did not differ significantly between the groups. 